\title{
Erratum: Spatial analysis of groundwater potential using remote sensing and GIS-based multi-criteria evaluation in Raya Valley, northern Ethiopia
}

\author{
Ayele Almaw Fenta $\cdot$ Addis Kifle • \\ Tesfamichael Gebreyohannes • Gebrerufael Hailu
}

\section{Erratum to: Hydrogeology Journal (2014) \\ DOI 10.1007/s10040-014-1198-x}

Part of Table 4 was missing from the original article; the full version is given below. Table 8 of the original article presented the correct and full data but in an awkward fashion, so it is also reproduced here.

Table 4 Pair-wise comparison matrix for lineament density $\left(\mathrm{km} / \mathrm{km}^{2}\right)$ classes $(\mathrm{CR}=0.05)$

\begin{tabular}{lllllll}
\hline & $4.2-6.5$ & $3.9-<4.2$ & $2.6-<3.9$ & $1.3-<2.6$ & $0-<1.3$ & Rate \\
\hline $4.2-6.5$ & 1 & - & - & - & - & 0.49 \\
$3.9-<4.2$ & $1 / 3$ & 1 & - & - & - & 0.25 \\
$2.6-<3.9$ & $1 / 4$ & $1 / 3$ & 1 & - & - & 0.14 \\
$1.3-<2.6$ & $1 / 6$ & $1 / 4$ & $1 / 3$ & 1 & - & 0.08 \\
$0-<1.3$ & $1 / 9$ & $1 / 6$ & $1 / 4$ & $1 / 3$ & 1 & 0.04 \\
\hline
\end{tabular}

Table 8 Pair-wise comparison matrix for annual rainfall (mm) classes $(\mathrm{CR}=0.01)$

\begin{tabular}{lllllll}
\hline & $921-1,021$ & $821-<921$ & $721-<821$ & $621-<721$ & $521-<621$ & Rate \\
\hline $921-1,021$ & 1 & - & - & - & - & 0.50 \\
$821-<921$ & $1 / 3$ & 1 & - & - & - & 0.26 \\
$721-<821$ & $1 / 4$ & $1 / 3$ & 1 & - & - & 0.13 \\
$621-<721$ & $1 / 6$ & $1 / 4$ & $1 / 3$ & 1 & - & 0.07 \\
$521-<621$ & $1 / 9$ & $1 / 6$ & $1 / 4$ & $1 / 3$ & 1 & 0.04 \\
\hline
\end{tabular}

Published online: 4 December 2014

(C) Springer-Verlag Berlin Heidelberg 2014

The online version of the original article can be found at http:// dx.doi.org/10.1007/s10040-014-1198-x.

\section{A. A. Fenta (-)}

College of Dryland Agriculture and Natural Resources, Department of Land Resources Management and Environmental Protection, Mekelle University, P.O. Box 231, Mekelle, Tigray, Ethiopia

e-mail: almawayele@yahoo.com

\section{A. Kifle $\cdot$ G. Hailu}

Institute of Geo-information and Earth Observation Sciences, Mekelle University, P.O. Box 231, Mekelle, Tigray, Ethiopia

T. Gebreyohannes

College of Natural and Computational Sciences, Department of Earth Sciences,

Mekelle University, P.O. Box 231, Mekelle, Tigray, Ethiopia 